\title{
On the slow motion of a spheroid in a rotating stratified fluid
}

\author{
E. R. Johnson ${ }^{1}$ and Stefan G. Llewellyn Smith ${ }^{2}$ \\ ${ }^{1}$ Department of Mathematics, University College London, London WC1E 6BT, UK \\ ${ }^{2}$ Department of Mechanical and Aerospace Engineering, Jacobs School of Engineering, UCSD, \\ La Jolla CA 92093-0411, USA \\ (Received xx; revised xx; accepted xx)
}

We consider the slow motion generated when a body is set into motion relative to an incompressible, inviscid, non-diffusive rotating stratified fluid, showing that there is generated in general a topographic Rossby wave which leads to non-decaying fluctuations in the lift on the obstacle and a fluctuating non-zero drag. The problem is relevant to the flow patterns and forces excited when slow oceanic flows cross bottom topography and suggests a mechanism for slow fluctuations observed in laboratory experiments.

\section{Introduction}

This paper considers the slow motion generated when a body is set into motion relative to an incompressible, inviscid, non-diffusive fluid, rotating about a vertical axis with angular velocity $\frac{1}{2} f$, whose density is vertically stratified with buoyancy frequency $N$. The body is initially at rest relative to the fluid, which is in solid body rotation, and at time $t=0$ the body is set into horizontal motion in a straight line at speed $U_{0}(t)$. Relative to an observer moving with the body the surrounding fluid moves at speed $U_{0}$. The problem is related to the geophysical flow set up by a current flowing over topography on the bottom of the ocean (Huppert \& Bryan 1976).

Stewartson $(1953,1967)$ discusses the unstratified $(N=0)$ version in describing the role of inertial wave radiation in the setting up of the Taylor column observed in experiments of rapidly-rotating flow past an obstacle (Taylor 1923). Johnson (1984) shows that Stewartson's solution can be regarded as the linear version of the strongtopography limit of the barotropic quasigeostrophic equations. Direct consideration of this limit shows that, contrary to the assumption of Stewartson (1967), inviscid finitedepth flow does not approach a steady limit at large time. Undamped topographic Rossby waves cycle compact topography leading to oscillatory, non-decaying drag and lift on the obstacle. The force fluctuations introduced by these waves are clearly visible in the fullynonlinear numerical integrations of the equations of motion in James (1980). For deeper containers (Johnson 1982; Cheng \& Johnson 1982), outside the aspect ratio of typical oceanic flows, topographic waves are weaker, being absent entirely in the unbounded linear analysis of Stewartson (1953). The constraint of a horizontal upper boundary at height small compared to $L / R o$ (for typical horizontal scale $L$, speed $U$ and Rossby number $R o=U / f L)$ is necessary to provide the vortex compression to support Rossby waves in unstratified flow.

Density stratification can also constrain the flow vertically and Grimshaw (1969) describes the internal wave field generated in the present problem for non rotating flow $(f=0)$. The question tackled here is whether stable stratification can provide a sufficiently strong vertical constraint to allow topographic Rossby waves to be generated and thus lead to fluctuating lift (i.e. horizontal force perpendicular to the flow at large 


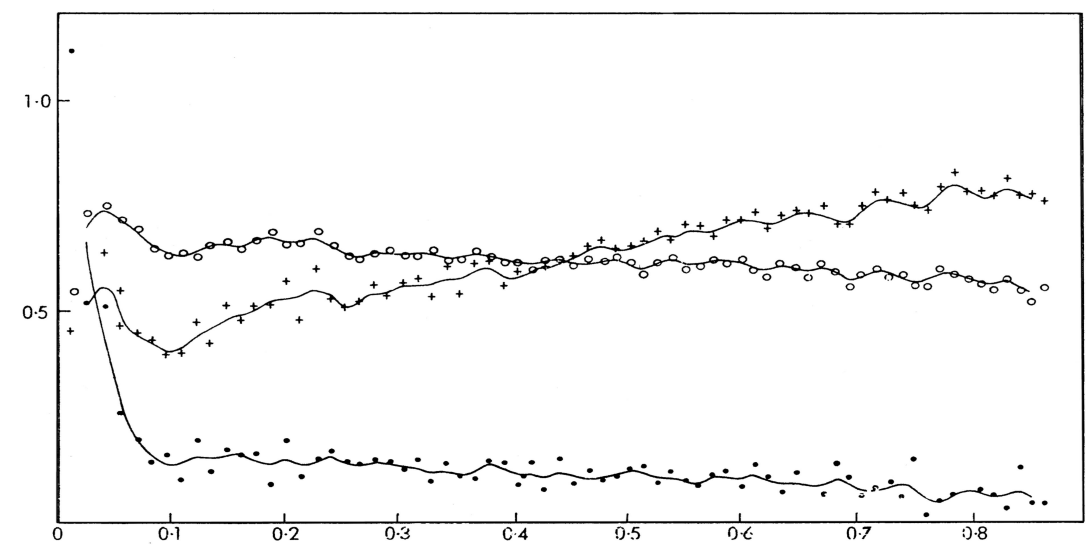

FiguRE 1. The non-dimensional drag and lift on a sphere of diameter $50.8 \mathrm{~mm}$ moving through a rotating, stratified fluid, with $N=1.6 \mathrm{~s}^{-1}$ and $f=3.8 \mathrm{~s}^{-1}$, plotted by Mason (1977) as a function of the distance in metres along the path of the sphere. The symbols give the actual measurements and the smooth curves are the result of applying a 1:2:1 filter to the data. In terms of the variables of $\S 4 ;+$ denotes the lift $-(3 / 4 \pi) \mathcal{F}_{y} ; \bullet$, the $\operatorname{drag}(3 / 4 \pi) \mathcal{F}_{x} ; \circ$, half the flow speed $\frac{1}{2} U \mathrm{~s}^{-1}$. Correlated fluctuations of distinct period and comparable magnitudes can be seen superposed on the mean values.

distances) and drag even in infinite-depth flow. It is shown below that this does indeed occur, and consequently the drag and lift in inviscid flow can have significant fluctuations for all time.

Few laboratory experiments in rotating flows have considered the forces on obstacles. d'Hieres, Davies \& Didelle (1990) report fluctuating forces on obstacles in unstratified rotating flow but at Rossby numbers sufficiently larger than those considered here that the flow is dominated by periodic vortex shedding. Mason (1977) reports force measurements in stratified rotating flow past a sphere, noting that his results become effectively independent of the fluid depth provided that the depth is greater than $2 \mathrm{fL} / \mathrm{N}$. Mason (1977) concentrates on average forces but his figure 5, reproduced here in figure 1 , shows fluctuations about the mean in both drag and lift. It is noted in $\S 5$ that these fluctuations are consistent with the topographic waves discussed here.

\section{Governing equations}

Consider an unbounded incompressible inviscid fluid rotating with uniform angular velocity $\frac{1}{2} f$ about a vertical axis $O z^{*}$. Take Cartesian axes $O x^{*} y^{*} z^{*}$ fixed in the rotating frame and let the density of the fluid $\bar{\rho}+\rho_{0}\left(z^{*}\right)+\rho^{*}\left(\boldsymbol{x}^{*}, t\right)$ be such that the buoyancy frequency $N=\left[-(g / \bar{\rho}) \mathrm{d} \rho_{0} / \mathrm{d} z^{*}\right]^{-1 / 2}$ is constant. A rigid neutrally $\dagger$ buoyant body with typical horizontal scale $L$ and surface $\mathcal{S}$ occupies a volume $\mathcal{V}$ surrounding the origin. For definiteness the body is taken to be set impulsively into motion at time $t^{*}=0$ so that it translates along the $O x^{*}$ axis at uniform speed $U$. Arbitrary start-ups can however be considered without difficulty. We expect topographic waves to be generated at start-up. These waves will have frequencies of order $f \times$ [obstacle height, $(h$ say $) /$ dynamical height]. In stratified flow the dynamical height is the smaller of the fluid depth and the Prandtl height $f L / N$. Thus in infinitely-deep flow the topographic waves have frequencies of order

$\dagger$ If the body is not neutrally buoyant, the flow pattern is unchanged but the body experiences a centrifugal force equal to the centrifugal force it would experience in free space minus the centrifugal force on the fluid displaced. A related result is derived below for the Coriolis force. 
$N h / L$, i.e. of non-dimensional frequency $B=N h / f L$. If $B$ is small, these waves are of much lower frequency than the inertial waves generated by the initial motion. One way of making $B$ small is to take $h / L$ small. This is the quasi-geostrophic limit of obstacles of small slope. Alternatively for a sphere where $h=L, B=N / f$ and $B$ small corresponds to weak stratification. When $B$ is large, it appears that there will be little energy at frequencies below the inertial frequency. Note the topographic waves can be suppressed by a start-up with significantly longer start-up time scale than the wave periods as shown in $§ 3.3$. In terms of the non-dimensional variables,

$$
\begin{gathered}
t=t^{*} / T, \quad(x, y, z)=\left(x^{*} / L, y^{*} / L, z^{*} / L\right), \quad(u, v, w)=\left(u^{*} / U, v^{*} / U, w^{*} / U\right), \\
p=\left(\bar{p}+g \int^{z} \rho_{0} \mathrm{~d} z^{*}+p^{*}\right) \frac{T}{\bar{\rho} U L}, \quad \sigma=\frac{g T \rho^{*}}{\bar{\rho} U} .
\end{gathered}
$$

Here $T$ is an arbitrary time scale which may be taken as $f^{-1}$ in the rotation-dominated regime with $N / f<1$ and as $N^{-1}$ in the stratification-dominated regime with $N / f>1$. The importance of advection relative to the set-up time of the flows considered here is then measured by the non-dimensional parameter $\tau=U T / L$, which is taken to be sufficiently small that advective terms are negligible, i.e. $\tau \ll 1$. In the rotation-dominated regime this is equivalent to small Rossby number, and in the stratification-dominated regime to $U / N L \ll 1$, a form of small Froude number flow. The governing equations can then be written

$$
\begin{array}{r}
v_{t}+f u=-p_{y}, \quad w_{t}=-p_{z}-\sigma, \\
\sigma_{t}-N^{2} w=0, \quad u_{x}+v_{y}+w_{z}=0,
\end{array}
$$

in which $f$ and $N$ are now dimensionless. The boundary conditions on the flow are the requirements that the flow be undisturbed sufficiently far from the body and that the body be impermeable, i.e. the velocity of the fluid normal to the body matches the normal velocity $U \hat{\boldsymbol{x}} \cdot \hat{\boldsymbol{n}}$ of the body, so for $t>0$,

$$
\boldsymbol{u} \rightarrow 0 \quad \text { as } \quad x^{2}+y^{2}+z^{2} \rightarrow \infty, \quad \boldsymbol{u} \cdot \hat{\boldsymbol{n}}=\hat{\boldsymbol{x}} \cdot \hat{\boldsymbol{n}} \quad \text { on } \quad \mathcal{S} .
$$

Note that $\mathcal{S}$ can be taken to be fixed to leading order since $\tau \ll 1$. Similarly, perturbations to the underlying density field caused by the moving body are of order $\tau \ll 1$ and so are negligible to leading order.

Introduce the Laplace transform in $t$ with parameter $s$. Denote transforms by overbars and then suppress the overbars. The governing equations become

$$
\begin{aligned}
& s u-f v=-p_{x}, \quad s v+f u=-p_{y}, \quad s w=-p_{z}-\sigma, \\
& s \sigma-N^{2} w=0, \quad u_{x}+v_{y}+w_{z}=0,
\end{aligned}
$$

with boundary conditions

$$
\boldsymbol{u} \rightarrow 0 \quad \text { as } \quad x^{2}+y^{2}+z^{2} \rightarrow \infty, \quad \boldsymbol{u} \cdot \hat{\boldsymbol{n}}=s^{-1} \hat{\boldsymbol{x}} \cdot \hat{\boldsymbol{n}} \quad \text { on } \quad \mathcal{S},
$$

the factor $s^{-1}$ being the transform of the constant speed at $t>0$. The momentum equations $(2.5 a)$ can be rearranged to give the horizontal components of velocity $u, v$ in terms of pressure alone as can the density and vertical momentum equations for $w$. Substituting the resulting velocity vector in the continuity equation in $(2.5 b)$ gives the field equation

$$
\left(N^{2}+s^{2}\right)\left(p_{x x}+p_{y y}\right)+\left(f^{2}+s^{2}\right) p_{z z}=0 \quad \text { outside } \mathcal{V} .
$$

Let the bounding rigid surface $\mathcal{S}$ be given by $F(x, y, z)=0$, so the inviscid condition of 
no normal flux through $\mathcal{S}$ becomes

$$
\begin{gathered}
u F_{x}+v F_{y}+w F_{z}=s^{-1} F_{x} \text { on } \mathcal{S} \\
\text { i.e. }-s\left[p_{x} F_{x}+p_{y} F_{y}+\frac{\left(f^{2}+s^{2}\right)}{\left(N^{2}+s^{2}\right)} p_{z} F_{z}\right]+f p_{x} F_{y}-f p_{y} F_{x}=\frac{\left(f^{2}+s^{2}\right)}{s} F_{x} \quad \text { on } \mathcal{S} \text {. }
\end{gathered}
$$

The problem can be expressed more concisely by introducing $Z=\left[\left(N^{2}+s^{2}\right) /\left(f^{2}+\right.\right.$ $\left.\left.s^{2}\right)\right]^{1 / 2} z$. In terms of the three-dimensional, scaled, gradient operator $\nabla_{3}=\hat{\boldsymbol{x}} \partial_{x}+\hat{\boldsymbol{y}} \partial_{y}+$ $\hat{z} \partial_{Z}$, the problem reduces to

$$
\begin{array}{r}
\nabla_{3}^{2}=\nabla_{3} \cdot\left(\nabla_{3} p\right)=0 \quad \text { outside } \quad \mathcal{V} \\
\left(-s \nabla_{3} p+f \nabla_{3} Z \wedge \nabla_{3} p\right) \cdot \nabla_{3} F=\left[\left(f^{2}+s^{2}\right) / s\right] \hat{\boldsymbol{x}} \cdot \nabla_{3} F \quad \text { on } \quad \mathcal{S} .
\end{array}
$$

The solutions take a particularly simple form when the body is axisymmetric about $O z$. Take polar co-ordinates $(x, y)=(r \cos \theta, r \sin \theta)$ and let the surface $\mathcal{S}$ be given by $G(r, Z)=0$. Look for solutions of the form $p(x, y, Z, s)=\Re\left\{q(r, Z) \mathrm{e}^{\mathrm{i} \theta}\right\}$. Then boundary condition (2.9) becomes

$$
s\left(q_{r} G_{r}+q_{Z} G_{Z}\right)+\mathrm{i} f r^{-1} G_{r} q=-\left[\left(f^{2}+s^{2}\right) / s\right] G_{r} \quad \text { on } \quad \mathcal{S} .
$$

Introducing the gradient operator in the vertical plane, $\nabla_{V}=\hat{\boldsymbol{r}} \partial_{r}+\hat{Z} \partial_{Z}$, allows the boundary condition (2.10) to be written

$$
\left[s \nabla_{V} q+\mathrm{i} f q \nabla_{V}(\log r)\right] \cdot \nabla_{V} G=-\left[\left(f^{2}+s^{2}\right) / s\right] \nabla_{V} r \cdot \nabla_{V} G \quad \text { on } \quad \mathcal{S} .
$$

This form of the boundary condition is invariant under conformal mappings in the $(r, Z)$ plane.

\section{Spheroidal bodies}

\subsection{The pressure field}

As a straightforward example consider a spheroidal body with symmetry axis vertical. Let the horizontal radius of the body be $L$ and the vertical semi-axis have length $h=\alpha L$. The spheroid is oblate if $\alpha<1$ and prolate for $\alpha>1$. In terms of $Z$ the equation for $\mathcal{S}$ is

$$
G=x^{2}+y^{2}+(z / \alpha)^{2}-1=r^{2}+(Z / \beta)^{2}-1=0 \quad \text { on } \mathcal{S},
$$

where $\beta=\alpha\left[\left(N^{2}+s^{2}\right) /\left(f^{2}+s^{2}\right)\right]^{1 / 2}$. The scaling in moving from $(x, y, z)$ to $(x, y, Z)$ means that $\mathcal{S}$, although remaining a spheroid in the new co-ordinates, is not necessarily oblate in terms of $(x, y, Z)$ when it is oblate in terms of $(x, y, z)$. The analysis here is presented for $\mathcal{S}$ oblate in terms of $(x, y, Z)$ and the differences for $\mathcal{S}$ prolate sketched afterwards.

Consider $\beta<1$. Note that for small $s, \beta$ is almost independent of $s$ and so too is the transformed shape of $\mathcal{S}$. Introduce elliptic co-ordinates $(\xi, \eta)$ through $(r, Z)=$ $(a \cosh \xi \cos \eta, a \sinh \xi \sin \eta)$. Then $\mathcal{S}$ becomes the surface $\xi=\xi_{0}$ provided $a \cosh \xi_{0}=$ $1, a \sinh \xi_{0}=\beta$, so $a=\sqrt{1-\beta^{2}}, \xi_{0}=\tanh ^{-1} \beta, \sinh \xi_{0}=\beta / \sqrt{1-\beta^{2}}, \cosh \xi_{0}=$ $1 / \sqrt{1-\beta^{2}}$ and $\tanh \xi_{0}=\beta$. The co-ordinates $(\xi, \eta, \theta)$ form a standard oblate spheroidal system. The transformation from $(r, Z)$ to $(\xi, \eta)$ is conformal and so boundary condition (2.11) becomes (since $\nabla_{V} G$ is in the direction $\hat{\boldsymbol{\xi}}$ ) simply

$$
s q_{\xi}+\mathrm{i} f \beta q=-\beta\left[\left(f^{2}+s^{2}\right) / s\right] \cos \eta, \quad \text { on } \quad \xi=\xi_{0} .
$$

The coefficient of $q$ is independent of $\eta$ since $\log r$ is the sum of functions of $\xi$ and $\eta$ 
separately. The general separated solution of (2.9) of the appropriate form that remains bounded at $\eta= \pm \frac{1}{2} \pi$ and decays for large $|\xi|$ is

$$
q=\sum_{n=1}^{\infty} a_{n} P_{n}^{1}(\sin \eta) Q_{n}^{1}(\mathrm{i} \sinh \xi)
$$

where $P_{n}^{1}$ and $Q_{n}^{1}$ are the Associated Legendre functions of order 1 and degree $n$ of the first and second kinds (Morse \& Feshbach 1953). Now $P_{1}^{1}(z)=\sqrt{1-z^{2}}$, so $a_{n}=0$ for $n \neq 1$ and $a_{1}$ is determined explicitly in simple terms of surds of $\beta$ and thus $s^{2}$ through

$$
a_{1}\left[s \cosh \xi_{0} Q_{1}^{1 \prime}\left(\mathrm{i} \sinh \xi_{0}\right)+f \beta Q_{1}^{1}\left(\mathrm{i} \sinh \xi_{0}\right)\right]=\mathrm{i} \beta\left(f^{2}+s^{2}\right) / s .
$$

\subsection{The force on the spheroid}

As the pressure is even in $z$ the total hydrodynamic force on the spheroid can be written $\mathcal{F}=F^{x} \hat{\boldsymbol{x}}+F^{y} \hat{\boldsymbol{y}}$ and the Laplace transform of the $x$-component as

$$
\bar{F}^{x}=-2 \int_{\mathcal{S}^{+}} p \hat{\boldsymbol{x}} \cdot \hat{\boldsymbol{n}} \mathrm{d} S=-2 \int_{\mathcal{R}} p_{\mathcal{S}^{+}} \frac{\hat{\boldsymbol{x}} \cdot \nabla G}{\hat{\boldsymbol{z}} \cdot \nabla G} r \mathrm{~d} r \mathrm{~d} \theta=-2 \pi \alpha^{2} \int_{r=0}^{1} \Re\left\{q_{\mathcal{S}^{+}}\right\}\left(r^{2} / z\right) \mathrm{d} r,
$$

where $\mathcal{S}^{+}$is the upper half of the body surface and $\mathcal{R}$ is the projection of $\mathcal{S}^{+}$on $z=0$, i.e. the unit disc $0 \leqslant r \leqslant 1$. The $y$-component of force can be expressed similarly, giving the combined complex force

$$
\bar{F}^{x}-\mathrm{i} \bar{F}^{y}=-2 \pi \alpha^{2} \int_{r=0}^{1} q_{\mathcal{S}^{+}}\left(r^{2} / z\right) \mathrm{d} r=-2 \pi \alpha \beta \int_{r=0}^{1} q_{\mathcal{S}^{+}}\left(r^{2} / Z\right) \mathrm{d} r .
$$

Now on $\mathcal{S}^{+}, r=\cos \eta, Z=\beta \sin \eta$ and $q_{\mathcal{S}}=a_{1} r Q_{1}^{1}\left(\mathrm{i} \sinh \xi_{0}\right)$, so

$$
\begin{aligned}
\bar{F}^{x}-\mathrm{i} \bar{F}^{y} & =-2 \pi \alpha \beta a_{1} Q_{1}^{1}\left(\mathrm{i} \sinh \xi_{0}\right) \int_{\eta=\pi / 2}^{0}\left(\cos ^{3} \eta / \beta \sin \eta\right)(-\sin \eta) \mathrm{d} \eta, \\
& =-(4 \pi / 3) \mathrm{i} \alpha \beta\left(f^{2}+s^{2}\right) /[s(f \beta-\mathrm{i} s \gamma)],
\end{aligned}
$$

where $\gamma=\mathrm{i} \cosh \xi_{0} Q_{1}^{1 \prime}\left(\mathrm{i} \sinh \xi_{0}\right) / Q_{1}^{1}\left(\mathrm{i} \sinh \xi_{0}\right)$ and is given in terms of $\beta$ and thus $s$. It remains to invert the transform (3.8).

\subsection{Slow start-up and steady flow}

We can follow Lighthill (1965) and suppose that the body is set into motion with velocity $U \mathrm{e}^{\epsilon t} \hat{\boldsymbol{x}}$, with $0<\epsilon \ll 1$. At $t=-\infty$ the body is at rest and by $t=0$ the body is moving at velocity $U \hat{\boldsymbol{x}}$. It is sufficient to consider that part of the response proportional to $\mathrm{e}^{\epsilon t}$ since, relative to this term, other transients are exponentially small. This gives precisely the analysis above with $\epsilon$ replacing $s$ (and omitting the inverse power of $s$ on the right side of (2.6) and its derivatives). In the limit $\epsilon \rightarrow 0, \beta=\alpha S=B$ and (3.2) gives simply

$$
q=\mathrm{i} f \cos \eta \quad \text { on } \quad \xi=\xi_{0},
$$

where, here and below, $q$ is the un-Laplace-transformed variable. Thus (3.3) becomes

$$
q=\mathrm{if} \cos \eta Q_{1}^{1}(i \sinh \xi) / Q_{1}^{1}\left(\mathrm{i} \sinh \xi_{0}\right) .
$$

Equation $(2.5 b)$ shows that the flow is then in horizontal planes with $w=0$ identically. The force on the body reduces to a cross-stream horizontal 'lift' $(4 \pi / 3) \alpha$, independent of rotation and stratification (this is the mass of fluid displaced to the left of the direction of motion, independent of (linear) stratification), and zero drag as expected. Hence the 
flow is geostrophic, in horizontal planes around the spheroid. The flow is exactly that obtained if one simply assumes that the final state is steady, neglects all transients in the initial value problem and considers the pole at in the Laplace transform at $s=0$. The 'lift' force is equal and opposite to the Coriolis force on the spheroid of the same mass as that of the water displaced. Thus a neutrally buoyant spheroid experiences no net force: neither Coriolis nor centrifugal. In fact the result is completely general:

THEOREM 3.1. A neutrally buoyant body accelerated sufficiently slowly into uniform motion in a rotating stratified fluid experiences no net force.

Proof. In the limit $\epsilon \rightarrow 0$ the pressure satisfies

$$
p_{x x}+p_{y y}+(f / N)^{2} p_{z z}=0, \quad \text { with } \quad p=-f y \quad \text { on } \quad \mathcal{S} .
$$

Now a pressure field consistent with (3.11) is simply $p=-f y$, for which $\nabla p=-f \hat{\boldsymbol{y}}$. Hence the hydrodynamic force on the body is given by

$$
\mathcal{F}=-\int_{\mathcal{S}} p \hat{\boldsymbol{n}} \mathrm{d} \mathcal{S}=-\int_{\mathcal{V}} \nabla p=f \hat{\boldsymbol{y}} \int_{\mathcal{V}} \mathrm{d} \mathcal{V}=f|\mathcal{V}| \hat{\boldsymbol{y}}
$$

equal and opposite to the Coriolis force on the body.

\subsection{The slow mode}

Consider $\beta \ll 1$. Then in $(3.8) \gamma=-4 / \pi$, so

$$
\bar{F}^{x}-\mathrm{i} \bar{F}^{y}=-(4 \pi / 3) \mathrm{i} \alpha\left(f^{2}+s^{2}\right)\left[s^{-1}-(s-\mathrm{i} f \beta \pi / 4)^{-1}\right] .
$$

This expression has poles at $s=0$ and $s=$ if $\beta \pi / 4$. As expected, the second pole has $|s| \ll 1, \beta=B$, independent of $s$ and (3.13) can be inverted to give

$$
\bar{F}^{x}-\mathrm{i} \bar{F}^{y}=-(4 \pi / 3) \mathrm{i} \alpha f[1-\exp (\mathrm{i} f B \pi t / 4)] .
$$

The first term gives the Coriolis force on the fluid displaced by the spheroid and the second, of exactly the same magnitude, comes from a topographic wave cycling clockwise around the spheroid with period $T=8 / B$ which, since $B \ll 1$, is large compared to $f$, as expected. Since for this wave $s$ is of order $B, \beta=B+\mathcal{O}\left(B^{2}\right)$ and the expansion for $\gamma$ can be continued as $\gamma=-(4 / \pi)\{1+[(4 / \pi)-(\pi / 4)] B\}+\mathcal{O}\left(B^{2}\right)$, giving a topographic wave of period $T=(8 / f B)\{1+[(4 / \pi)-(\pi / 4)] B\}+\mathcal{O}(B)$. For $B=1 / 8$, this gives a period of approximately 68 .

The explicit form for the flow field is equally simple and consists of the steady flow and a clockwise rotating dipolar mode. Note that the vertical velocity is small, of order $B$, but non-zero. The horizontal velocity field is horizontally non-divergent (with streamfunction $p$ ) to leading order in $B$, but is horizontally divergent at first order.

\subsection{Strong stratification: the prolate case}

Consider $\beta>1$. Again, for small $s, \beta$ is almost independent of $s$ and so too is the transformed shape of $\mathcal{S}$. Introduce elliptic co-ordinates $(\xi, \eta)$ through $(r, Z)=$ $(a \sinh \xi \cos \eta, a \cosh \xi \sin \eta)$. Then $\mathcal{S}$ becomes the surface $\xi=\xi_{0}$ provided $a \sinh \xi_{0}=1$, $a \cosh \xi_{0}=\beta$, so $a=\sqrt{\beta^{2}-1}, \xi_{0}=\operatorname{coth}^{-1} \beta, \sinh \xi_{0}=1 / \sqrt{\beta^{2}-1}, \cosh \xi_{0}=$ $\beta / \sqrt{\beta^{2}-1}$ and $\operatorname{coth} \xi_{0}=\beta$. The co-ordinates $(\xi, \eta, \theta)$ form a standard prolate spheroidal system. Again, the transformation from $(r, Z)$ to $(\xi, \eta)$ is conformal and so boundary condition (2.11) becomes simply

$$
s q_{\xi}+\mathrm{i} f \beta q=-\beta\left[\left(f^{2}+s^{2}\right) / s\right] \cos \eta, \quad \text { on } \quad \xi=\xi_{0} .
$$


The general separated solution of (2.9) of the appropriate form that remains bounded at $\eta= \pm \frac{1}{2} \pi$ and decays for large $|\xi|$ is now

$$
q=\sum_{n=1}^{\infty} a_{n} P_{n}^{1}(\sin \eta) Q_{n}^{1}(\cosh \xi) .
$$

Again $a_{n}=0$ for $n \neq 1$ with $a_{1}$ given explicitly in simple terms of surds of $\beta$ and thus $s^{2}$ as

$$
a_{1}\left[s \sinh \xi_{0} Q_{1}^{1 \prime}\left(\cosh \xi_{0}\right)+\operatorname{if} \beta Q_{1}^{1}\left(\cosh \xi_{0}\right)\right]=-\beta\left(f^{2}+s^{2}\right) / s .
$$

The evaluation of the force components on the cylinder follows the oblate case except that now $q_{\mathcal{S}}=a_{1} r Q_{1}^{1}\left(\cosh \xi_{0}\right)$, giving the same final expression (3.8) with

$$
\gamma=\sinh \xi_{0} Q_{1}^{1 \prime}\left(\cosh \xi_{0}\right) / Q_{1}^{1}\left(\cosh \xi_{0}\right) .
$$

Combining the oblate and prolate cases gives

$$
\gamma= \begin{cases}\frac{2-\beta^{2}-\beta\left(\beta^{2}-1\right)^{-1 / 2} \tanh ^{-1}\left(\sqrt{\beta^{2}-1} / \beta\right)}{\beta-\left(\beta^{2}-1\right)^{-1 / 2} \tanh ^{-1}\left(\sqrt{\beta^{2}-1} / \beta\right)} & \text { if } \beta>1, \\ \frac{2-\beta^{2}-\beta\left(1-\beta^{2}\right)^{-1 / 2} \tan ^{-1}\left(\sqrt{1-\beta^{2}} / \beta\right)}{\beta-\left(1-\beta^{2}\right)^{-1 / 2} \tan ^{-1}\left(\sqrt{1-\beta^{2}} / \beta\right)} & \text { if } \beta<1,\end{cases}
$$

which also follows by noting that the expansion about the origin of $\tanh ^{-1}(z) / z$ contains only even powers of $z$. Note that for $\beta \gg 1,|\gamma| \sim \beta$ and so the pole in (3.8) at $s=$ if $\beta / \gamma$ simply gives a further contribution at the inertial frequency.

\section{Drag}

The Laplace transform of the complex force can be rewritten as

$$
\overline{\mathcal{F}}=-(4 \pi / 3) \mathrm{i} \alpha\left(f^{2}+s^{2}\right) /(s d),
$$

where $d(s)=f-\mathrm{i} s\left(2-\beta^{2}-t\right) /\left(\beta^{2}-t\right), t=\delta^{-1} \tan ^{-1} \delta$ and $\delta^{2}=1 / \beta^{2}-1$. The function $t$ is even in $\delta$ and has no branch point at $\delta=0$. The function $\mathcal{F}$ has branch points at $\pm \mathrm{i} N$ and $\pm \mathrm{i} f$, which come from $\beta$, and finite branch cuts along the imaginary axis from the smaller of these branch points in magnitude (possibly zero) to the larger. The pole at the origin leads to the contribution $\mathcal{F}_{0}=-(4 \pi / 3)$ i $\alpha f$, as discussed in $\S 3.3$.

The slow mode has real frequency $\omega_{*}$, corresponding to the simple pole at $s=\mathrm{i} \omega_{*}$, determined through $d\left(\mathrm{i} \omega_{*}\right)=0$. The physical system has a specific handedness because it combines rotation with a direction of translation, and $s=-\mathrm{i} \omega_{*}$ is not a pole. The slow mode is not related to the inertia-gravity wave spectrum. In particular it exists for $f=N$, when there are no inertia-gravity waves. In this case, the value of the slow-mode frequency can be found explicitly

$$
\omega_{*}=-f\left(\alpha^{2}-t\right) /\left(2-\alpha^{2}-t\right), \quad \delta^{2}=1 / \alpha^{2}-1 .
$$

For small $\alpha, \omega_{*} \sim(\pi / 4) f \alpha=(\pi / 4) N \alpha$, while for large $\alpha$, the frequency tends to $f=N$. For other values of $f$ and $N$, there is an inertia-gravity spectrum and $\omega_{*}$ lies between 0 and $\min (f, N)$. The contribution of the slow mode to the drag is given by

$$
\mathcal{F}_{*}=-(4 \pi / 3) \alpha \mathrm{e}^{\mathrm{i} \omega_{*} t}\left(f^{2}-\omega_{*}^{2}\right) /\left[\omega_{*} d^{\prime}\left(\mathrm{i} \omega_{*}\right)\right],
$$



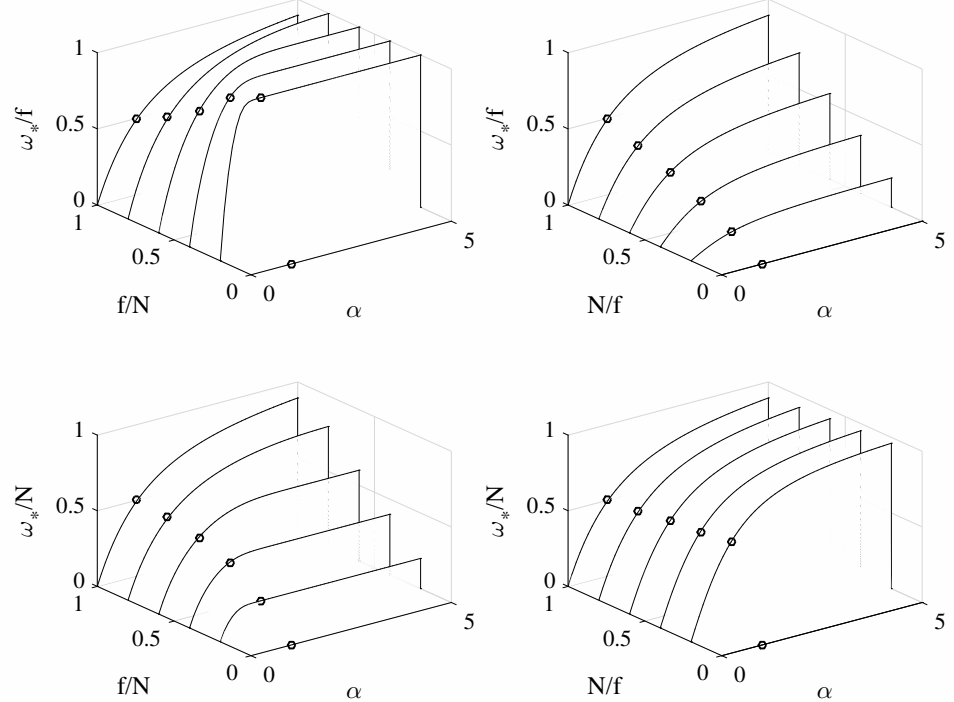

Figure 2. Frequency of the topographic mode. Left panels: $f \leqslant N$; right panels: $f \geqslant N$. Upper panels: non-dimensionalized by $f$; lower panels: non-dimensionalized by $N$. The value for a sphere $(\alpha=1)$ is indicated bv an onen circle.
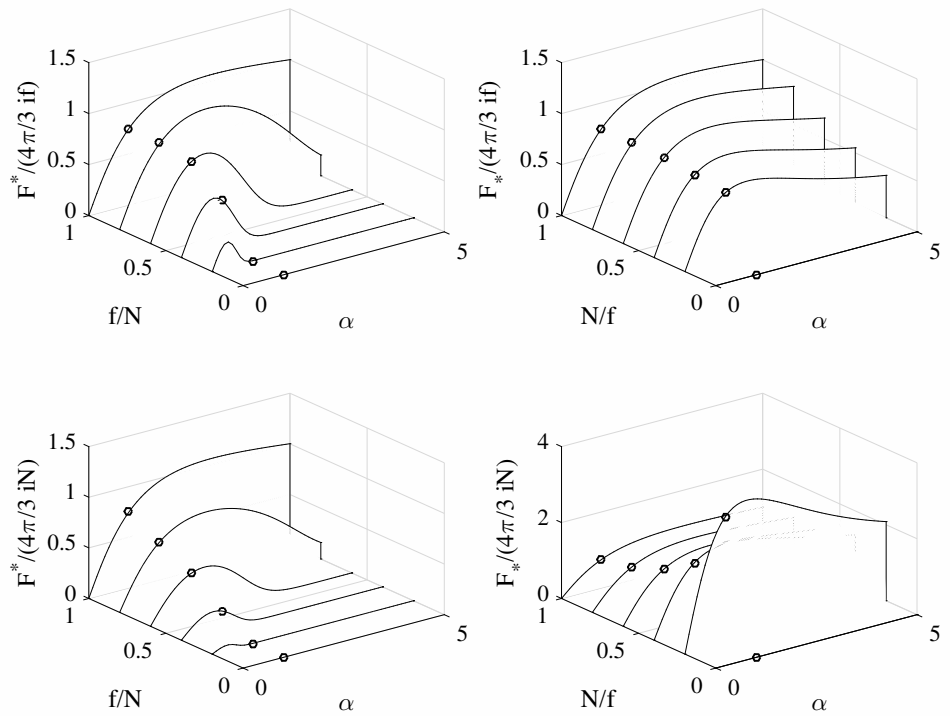

Figure 3 . As for figure 2 but for the force contribution from the topographic mode.

where

$d^{\prime}(s)=-\mathrm{i} \frac{2-\beta^{2}-t}{\beta^{2}-t}-2 \mathrm{i} \alpha^{2} s^{2} \frac{f^{2}-N^{2}}{\left(f^{2}+s^{2}\right)^{2}}\left[\frac{-2+2 t}{\left(\beta^{2}-t\right)^{2}}-\frac{1}{2 \beta^{4} \delta^{2}}\left(-t+\frac{1}{1+\delta^{2}}\right) \frac{2-2 \beta^{2}}{\left(\beta^{2}-t\right)^{2}}\right]$.

Figure 2 shows the frequency $\omega_{*}$ of the slow mode as a function of $N / f$ or $f / N$ and of $\alpha$. The frequency is non-dimensionalized using $f$ and $N$ separately to show the different limits. For large $\alpha$ it approaches $\min (N, f)$. Figure 3 shows the contribution to the force from the slow mode as a function of $N / f$ or $f / N$ and of $\alpha$, again non-dimensionalized 
using $N$ and $f$ separately. For $N \gg f$, the force contribution decays dramatically for large $\alpha$.

The inertia-gravity spectrum is dominated by the contributions from the branch points at $\pm \mathrm{i} f$ and $\pm \mathrm{i} N$. Expanding $\overline{\mathcal{F}}$ leads to

$$
\overline{\mathcal{F}} \sim\left(\pi^{2} / 3\right) \mathrm{e}^{3 \mathrm{i} \pi / 4} \alpha^{2} N^{-3 / 2} \sqrt{2\left(N^{2}-f^{2}\right)}(s-\mathrm{i} N)^{1 / 2} \quad \text { as } s \rightarrow \mathrm{i} N .
$$

A similar result holds for $s \rightarrow-\mathrm{i} N$. Combining the two leads to large-time behaviour of the form

$$
\mathcal{F}_{N}=-2 \pi^{5 / 2}(N t)^{-3 / 2} \mathrm{i} \alpha^{2} \sqrt{2\left(N^{2}-f^{2}\right)} \cos (N t+3 \pi / 4) \quad \text { as } t \rightarrow \infty .
$$

This is eventually smaller than the response of the slow mode, even though this may take a long time if the latter is small, which is the case for large $\alpha$ and small $f / N$, as can be seen in Figure 3.

The inertial contribution is more complicated:

$$
\overline{\mathcal{F}} \sim-\frac{4 \pi \alpha^{3}\left(N^{2}-f^{2}\right)}{3 f^{2} \log A(f+\mathrm{i} s)} \quad \text { as } s \rightarrow \text { if } \quad \text { with } A=\frac{f \mathrm{e}^{\alpha^{2}\left(N^{2}-f^{2}\right) / 2 f^{2}+2}}{2 \alpha^{2}\left(N^{2}-f^{2}\right)} .
$$

The asymptotic behaviour of this expression is related to Ramanujan's integral and can be shown to take the form $t^{-1 / 2} \mathrm{e}^{\mathrm{i} f t} \log \left(A^{-1} t\right)$ using e.g. the approach of Llewellyn Smith (2000). The singularity as $s \rightarrow-$ if contains a terms that behaves as $(s+\mathrm{i} f)^{2}$ times the logarithmic factor above, which leads to $t^{-5 / 2} \mathrm{e}^{-\mathrm{i} f t} \log \left(A^{-1} t\right)$ in the time domain. Once again, even though these terms decay, they can dominate the eventual slow mode response if the amplitude of the latter is small. We shall not pursue the details.

We now consider the cases of flat and steep obstacles to obtain the limiting values of the slow-mode frequency. For flat obstacles $(\alpha \ll 1), \omega_{*} \sim(\pi / 4) \alpha N$ as noted above: the topographic mode frequency scales with the buoyancy frequency. For steep obstacles $(\alpha \gg 1)$, the nature of the topographic response depends crucially on whether $N$ is larger or smaller than $f$. For $N<f$, which is not so interesting geophysically, $\omega_{*}^{2} \sim$ $N^{2}\left(1-\alpha^{-2} \xi^{2}\right)$, where $\xi$ satisfies $d=0$ with $\beta^{2}$ replaced by $\xi^{2} N^{2} /\left(f^{2}-N^{2}\right)$. Hence as the spheroid becomes steeper, the topographic frequency approaches the buoyancy frequency, which is smaller than the inertial frequency. For $f<N$, the appropriate scaling is $\omega_{*}^{2} \sim f^{2}\left(1-\sigma^{2}\right)$, where now

$$
\sigma \sim 2 \alpha\left(N^{2} / f^{2}-1\right)^{1 / 2} \exp \left[-\left(\alpha^{2} / 4\right)\left(N^{2} / f^{2}-1\right)-1\right]
$$

so the frequency becomes exponentially close to the inertial frequency. In addition, the factor $\left(f^{2}+s^{2}\right)$ in $\overline{\mathcal{F}}$ shows that the amplitude of the topographic mode is exponentially small in $\alpha$ provided $N$ is not too close to $f$. Distinguished limits arise when $N \sim f$, but again will not be pursued here.

\section{Discussion}

We have considered the slow motion generated when a body is set into motion relative to an incompressible, inviscid, non-diffusive rotating stratified fluid, showing that there is present in general a topographic Rossby wave which leads to non-decaying fluctuations in the lift on the obstacle and a fluctuating non-zero drag. Some special cases deserve mention. If $f=N=0$, the flow here reduces to steady three-dimensional potential flow past a spheroid. If $f=0$, we recover the case considered by Grimshaw (1969): the system supports internal waves and evolves to the ultimate steady flow discussed by Drazin (1961). There is no topographic mode. If $N=0$, the branch cut of $\S 4$ extends 
from - if to if, leaving no space for a pole on the imaginary axis between the origin and the inertial-wave branch point, so there is no longer a slow mode and we have the problem and result of Stewartson (1953). This latter solution is only a local solution and, as pointed out by Lighthill (Hide, Ibbetson \& Lighthill 1968), at large heights the disturbance decomposes into an inertial wave field (Cheng \& Johnson 1982). The strength of the slow mode depends on the abruptness of the initiation of the motion and it has been shown that for sufficiently slowly accelerated bodies the mode can be arbitrarily weak.

The observations of Mason (1977) reproduced in figure 1 can now be discussed in terms of the results above. We digitised the data from the published curves. The steady mean term $\mathcal{F}_{0}$ gives zero mean drag and lift, scaled as plotted, of unity. As Mason notes, the observations are in reasonable accord with these, with deviations ascribable to viscosity and asymmetries introduced by weak nonlinearity, since $R o \sim 0.12$ here. The fluctuations are small as the sphere in the experiment accelerates smoothly from rest. The sampling frequency can be recovered by dividing the differences in path values by the observed velocity, and is $1 \mathrm{~Hz}$ to within $3 \%$. This does not resolve $f\left(3.8 \mathrm{rad} \mathrm{s}^{-1}\right)$. We used the last 40 seconds of data to avoid early-time effects. A least-squares fit to obtain the dominant frequencies in the data showed periods close to $N\left(1.6 \mathrm{rad} \mathrm{s}^{-1}\right)$ and to the aliased values of $f$ and $2 f$ within the resolved frequencies (2.48 and $1.32 \mathrm{rad} \mathrm{s}^{-1}$ respectively). The lift curve also showed a signal with frequency of approximately $0.87 \mathrm{rad} \mathrm{s}^{-1}$. For the experimental values of $\alpha=1$ and $N / f=0.42$, the slow mode has predicted frequency $\omega_{*}=0.9 \mathrm{rad} \mathrm{s}^{-1}$, lending support to the suggestion that the observed fluctuations are due to the topographic Rossby wave, despite the limitations of the data. In particular, transients and the inertia-gravity band are clearly visible in the data. For a slow mode cycling topography with shallow fluid to its right, Johnson (1984), for unstratified flow, and (4.3) for stratified flow, show that fluctuations in the lift should lag those in the drag by $\pi / 2$. The data show that the fluctuations in lift and drag are not in phase but are insufficiently detailed to derive phase information. Experiments (possibly numerical) with better sampling could confirm the presence of the topographic mode at large times.

\section{REFERENCES}

Cheng, H. K. \& Johnson, E. R. 1982 Inertial waves above an obstacle in an unbounded, rapidly rotating fluid. Proc. $R$. Soc. London A 383, 71-87.

D'Hieres, G. C., Davies, P. A. \& Didelle, H. 1990 Experimental studies of lift and drag forces upon cylindrical obstacles in homogeneous, rapidly rotating fluids. Dyn. Atmos. Oceans 15, 87-116.

Drazin, P. G. 1961 On the steady flow of a fluid of variable density past an obstacle. Tellus 13, 239-251.

Grimshaw, R. 1969 Slow time-dependent motion of a hemisphere in a stratified fluid. Mathematika 16, 231-248.

Hide, R., Ibbetson, A. \& Lighthill, M. J. 1968 On slow transverse flow past obstacles in a rapidly rotating fluid. J. Fluid Mech. 32, 251-272.

Huppert, H. E. \& Bryan, K. 1976 Topographically generated eddies. Deep-Sea Res. 23, 655679.

JAmes, I. N. 1980 The forces due to geostrophic flow over shallow topography. Geophys. Astrophys. Fluid Dyn. 14, 225-250.

Johnson, E. R. 1982 The effects of obstacle shape and viscosity in deep rotating flow over finite-height topography. J. Fluid Mech. 120, 359-383.

Johnson, E. R. 1984 Starting flow for an obstacle moving transversely in a rapidly rotating fluid. J. Fluid Mech. 149, 71-88.

Lighthill, M. J. 1965 Group velocity. J. Inst. Maths Applics 1, 1-28. 
Llewellyn Smith, S. G. 2000 The asymptotic behaviour of Ramanujan's integral and its application to two-dimensional diffusion-like equations. Eur. J. Appl. Math. 11, 13-28.

Mason, P.J. 1977 Forces on spheres moving horizontally in a rotating stratified fluid. Geophys. Astrophys. Fluid Dyn. 8, 137-154.

Morse, P. M. \& Feshbach, H. 1953 Methods of Theoretical Physics. New York, NY: McGrawHill.

Stewartson, K. 1953 On the slow motion of an ellipsoid in a rotating fluid. Q. J. Mech. Appl. Math. 6, 141-162.

Stewartson, K. 1967 On slow transverse motion of a sphere through a rotating fluid. J. Fluid Mech. 30, 357-369.

TAYlor, G. I. 1923 Experiments on the motion of solid bodies in rotating fluids. Proc. R. Soc. London A 104, 213-218. 